\title{
Antigen-Specific Therapy of Multiple Sclerosis: The Long- Sought Magic Bullet
}

\author{
Lawrence Steinman \\ Department of Neurology and Neurological Sciences, Stanford University, Stanford, CA 94305
}

\begin{abstract}
Summary: The adaptive immune response in multiple sclerosis (MS) targets various myelin proteins and even some inducible heat shock proteins. A few attempts have been made to tolerize relapsing-remitting patients with MS to either fulllength myelin basic protein or to a key peptide epitope between residues 83-99. These trials have demonstrated that this approach may potentially provide benefit to patients with relapsing- remitting MS. However, manipulation of responses to myelin proteins can have deleterious effects. The immune re-
\end{abstract}

sponse to myelin components is positioned at a key tipping point in the pathophysiology of the disease. Clarification of the key target antigens in MS, and better understanding of practical methods to attain tolerance to a wide variety of myelin and neuronal molecules will provide the basis for the ultimately successful antigen specific therapy. Key Words: Multiple sclerosis, altered peptide ligand, DNA vaccine, myelin basic protein, magic bullet.

\section{INTRODUCTION}

Multiple sclerosis (MS) is an organ specific disease involving perivascular lymphocytic infiltration, leading to inflammation of white matter and subsequent degeneration of both white matter and underlying axons. ${ }^{1,2}$ Adaptive immune responses, generated from antigen specific $T$ cells and from antibody producing $B$ cells, have been described in the blood, spinal fluid and central nervous system (CNS) of patients with MS. Indeed there are a large number of specific protein and lipid antigens targeted by the immune system's $\mathrm{T}$ cells and antibody producing B cells. Currently, there are no approved therapies aimed at modulating these adaptive immune responses in MS, though there is evidence from pre-clinical work in the mouse that one of the current approved therapies for MS, glatiramer, may influence adaptive immunity indirectly via modulation of type 2 monocytes. $^{3}$

Instead of targeting adaptive immunity, current therapies for MS are directed at modulating, often actually deleting, very broad swathes of the immune system. When a large chunk of the immune system is deleted or "blocked", though benefits may be apparent, the risks are

Address correspondence and reprint requests to: Lawrence Steinman, MD, Dept. of Neurology and Neurological Sciences, Stanford University, Stanford, CA 94305. E-mail: steinman@stanford.edu. stark. Experience has taught us from work on immune suppression for tolerance of allograft transplants, that physicians cannot remove huge chunks of our antibodyproducing armor without running the risk of serious and fatal opportunistic infections.

For example, therapies of autoimmune diseases aimed at blocking CD20 or the integrin alpha4 beta 1 have lead to fatal opportunistic infections in patients with MS, Crohn's Disease, and systemic lupus erythematosus. Thus, indiscriminately killing all B cells with monoclonal antibodies targeting CD20, or blocking the homing of all monocytes, T cells, B cells and macrophages to the CNS, with monoclonal antibodies binding to the adhesion molecule alpha 4 beta 1 integrin, have led to devastating infections with progressive, multifocal leukoencephalopathy, known as PML.,

In contrast to approaches that aim at modulation of the immune system even with highly selective monoclonal antibodies that block or delete one of its fundamental components, the hope is that in MS and in other immune diseases, tolerization of particular adaptive immune responses will preserve the ability of the immune system to fight infections while deleting the immune system's proclivity for attacking its self-constituents. Antigen specific treatment, that might be hailed as a "magic bullet, which reduces only those immune responses that are involved in an autoimmune disease while leaving the rest of the 
immune system intact, has so far evaded medical researchers. Remarkably few trials have been undertaken in humans with these antigen specific approaches to diseases like MS, even though a large number of pre-clinical experiments indicate that such a strategy might indeed work in man. Here I shall review what so far has been attempted in man, and the challenges that we face in devising such a therapy that would be truly worthy of the title that I think Paul Ehrlich intended, when he first unveiled the term "magic bullet".

In this article I shall explore four ongoing examples where investigators are attempting antigen specific approaches for the treatment of MS: two trials with the same altered peptide ligand directed to the 83-99 peptide of myelin basic protein ${ }^{8,9}$; a trial of therapy with a peptide to myelin basic protein $82-98^{10}$; and a trial of a DNA vaccine directed to the entire myelin basic protein molecule. ${ }^{11}$ Earlier attempts by Jonas Salk and colleagues to tolerize the immune system with porcine myelin basic protein, ${ }^{12,13}$ and by Weiner and colleagues ${ }^{14}$ to tolerize via orally delivering myelin antigens will be discussed in context.

In a recent editorial entitled, "The Coming of Age for Antigen Specific Therapy of MS," I wrote,

There is a compelling need for novel approaches for treatment of MS. Though there are some approved therapies for multiple sclerosis, the efficacy of these current treatments leaves ample room for improvement. For example the data from the pivotal trials leading to the approval of the beta interferons and glatiramer acetate revealed that relapse rates were reduced by about a third, in patients with relapsing remitting multiple sclerosis [MS]. Recent attempts to treat MS with blockade of cytokines like TNF, though highly successful in diseases like rheumatoid arthritis and Crohn's Disease, have actually produced a worsening of MS, leading to the requirement for warning labels from European and American regulatory agencies. Approaches aimed at blocking lymphocyte homing, have had a bipolar existence. The alpha4 integrin blocker, Tysabri, doubled the beneficial effect of the drug on relapse rate, compared to those currently approved, reducing relapse rates by two thirds. However, Tysabri has a small but finite risk of about one per thousand, for serious and sometimes fatal opportunistic infections, like progressive multifocal leuko-encephalopathy. Ultimately, a successful immune therapy for MS would involve identification of the major immune responses targeting the central nervous system in the disease, and then shutting down or 'tolerizing' the immune system so that pathogenic autoimmunity is not occurring. ${ }^{15}$
TABLE 1. Similarities in Sequence of Microbes and a Key Region of Myelin Basic Protein

\begin{tabular}{|c|c|}
\hline Peptide & Sequence ${ }^{\mathrm{a}}$ \\
\hline MBP peptide & ENPVVHFFKNIVTP \\
\hline \multicolumn{2}{|l|}{ Viral peptides } \\
\hline Human papillomavirus 7 & IGGRVHFFKDISPIA \\
\hline Human papillomavirus 13 & IGGR $\overline{\text { VHFFKDISPIS }}$ \\
\hline Human papillomavirus 40 & IGGRVHFFRDISPIG \\
\hline Cytomegalovirus & DRHPVYFFKSACPPN \\
\hline Dhori virus & SDDFIHFFKA KSYDD \\
\hline Herpes simplex virus type 1 & GGRRLFFVKAHVRES \\
\hline Epstein-Barr virus & TGGVY $\overline{\text { HFVKKKHVHES }}$ \\
\hline Influenza A & KDMTKEFFKNKSETW \\
\hline Epstein-Barr virus & VSGFISFFKNPFGGM \\
\hline Hepatitis A virus & EVKPASFFKNPHNDM \\
\hline Human adenovirus & LATYHIFFKNQRIPL \\
\hline
\end{tabular}

$\mathrm{MBP}=$ myelin basic protein.

${ }^{a}$ Underscoring highlights the region of similarity between MBP and viral peptide sequences.

\section{THE ANTIGENIC TARGETS OF T CELLS AND ANTIBODIES IN MS}

There is ample evidence that the strongest adaptive immune response in MS, at both the T cell and antibody level, is directed not to a myelin protein, but to an induced small heat shock protein, aB crystallin $(\mathrm{aBC}){ }^{16,17}$ This small heat shock protein is the most abundant transcript that is unique to the MS lesion, meaning that it is not found in normal brain. ${ }^{18}$ aBC serves as a brake on many aspects of the inflammatory process. Its presence reduces pro-inflammatory cytokine production, including Th1 and Th17 cytokines, inhibits phosphorylation of p38 map kinase, and reduces apoptosis, by inhibiting the cleavage of caspase- $3 .{ }^{17} \mathrm{~T}$ cell responses and antibodies are also directed against all the major myelin proteins in MS including myelin basic protein (MBP), proteolipid protein (PLP)], myelin oligodendroglial glycoprotein (MOG), myelin associated glycoprotein (MAG), and MOBP. ${ }^{17,19}$ Other protein components in the myelin sheath such as transaldolase and CNPase are targets of the immune response in MS. ${ }^{19,20}$ Lipids and carbohydrates of the myelin sheath are also targeted in MS. ${ }^{21}$

The immune response to one region of myelin basic protein has been studied the most intensively. The peptide between residues 82 and 99 on myelin basic protein is a major target of both $\mathrm{T}$ and $\mathrm{B}$ cell responses to MBP. ${ }^{18}$ Modulating the immune response to this region on myelin basic protein has been the subject of attempts at immune therapy in MS, with both altered peptides ligands and native protein. This region of MBP has homology to similar peptides that are found in various microbes (Table 1), ${ }^{22-24}$ In theory tolerizing to this epitope could lead to tolerization against the corresponding microbe, but fortunately the adaptive immune re- 
sponse to microbes may be far more diverse than it is to self-antigens.

\section{TRIALS WITH ALTERED PEPTIDE LIGANDS IN MULTIPLE SCLEROSIS}

An altered peptide ligand (APL) to the region of myelin basic protein 83-99 was devised. The basic concept behind the first generation of APLs was to maintain binding to HLA class II molecules, while modulating T cell receptor signaling so that instead of a pro-inflammatory $\mathrm{T}$ helper 1 response, a counteractive $\mathrm{T}$ helper 2 response would be mounted. By mutating the key $\mathrm{T}$ cell receptor contact site and changing it from lysine to alanine, and by preserving the key contact residues for binding to HLA class II, signaling through the T cell receptor would be modulated. This approach was consummated with great success in various rodent models of multiple sclerosis, collectively referred to as experimental autoimmune encephalomyelitis. In pre-clinical experiments this altered peptide ligand with altered lysine to alanine was highly effective in both preventing and reversing experimental autoimmune encephalomyelitis. $^{25-27}$ The APL was capable of reducing antigen specific immune responses associated with release of gamma interferon and with TNF. When this peptide was taken forward into humans in Phase 1 and Phase 2 clinical trials in relapsing remitting MS patients, antigen specific $\mathrm{T}$ cell responses to native and altered myelin basic protein peptide 82-98 were observed. These responses had characteristics of a $\mathrm{T}$ helper 2 type associated with production of IL-5. ${ }^{9,28}$ In some patients such T helper 2 responses to both myelin basic protein and to its altered peptide ligand were monitored four years after cessation of therapy with the APL. ${ }^{29}$

In an open label trial in eight patients with MS at the National Institutes of Health there was overall no clinical worsening in clinical or MRI parameters. ${ }^{8}$ However, two patients receiving weekly $50 \mathrm{mg}$ doses of this APL had exacerbations of disease. In these two patients there was an increase in $\mathrm{T}$ helper 1 type responses with a large increase in frequency in $T$ cells responding to both the altered peptide ligand and native peptide, 83-99 of myelin basic protein. ${ }^{8}$ One patient had been monitored frequently with over 100 MRI scans over the preceding nine years. This patient had never shown more than 30 lesions on any of the 100 scans. However, after the fifth $50 \mathrm{mg}$ injection of the APL, this patient had 91 contrast enhancing lesions. A second patient receiving the $50 \mathrm{mg}$ doses reported the "disappearance of her chronic symptoms" during the first 5 months of APL therapy. Her clinical and MRI measures improved during the first 5 months. At that point the patient developed a right parieto-occipital lesion, with a correlative left superior homonymous quadrant anopia. ${ }^{8}$
A third patient with atypical MS, who had a demyelinative polyneuropathy, had an exacerbation at the $5 \mathrm{mg}$ per week dose of the APL. Interestingly, whereas MBP reactive $\mathrm{T}$ cells rose in the two patients receiving the 50 $\mathrm{mg}$ dose of APL, MBP reactive cells disappeared from the patient who received the $5 \mathrm{mg}$ dose. All patients returned to baseline after standard i.v. steroid therapy for their relapses. These experiments indicated that at high doses of APL a rise in MBP reactive $\mathrm{T}$ cells of the T helper 1 phenotype, associated with relapse, was observed. There may have been a causal link to administration of the APL. ${ }^{8}$

In contrast to the small study performed at the National Institutes of Health, a placebo-controlled, randomized double-blind trial was undertaken with the same APL. ${ }^{9}$

The trial was terminated prematurely after 13 patients; approximately $9 \%$ of those dosed developed immediate hypersensitivity reactions. Such reactions were manifest by signs and symptoms including injection site erythema, itching, paresthesias, exanthomatous rash, dyspnea, and in a few cases urticaria. Almost all of the reactions occurred after 10 or more weekly injections. These immediate hypersensitivity reactions were not accompanied by worsening of disease. ${ }^{9}$

In those patients who received all 16 weekly injections there was evidence of a decrease in contrast enhancing lesions and a reduction in lesion volume in those individuals receiving the $5 \mathrm{mg}$ weekly dose. In addition there was evidence of a $\mathrm{T}$ helper 2 response to both the APL and to the native myelin basic protein, ${ }^{9}$ In a subsequent trial, dosing frequency was reduced and neither immediate hypersensitivity reactions, nor evidence of a change in magnetic resonance lesion activity was noted.

Lessons learned thus far from clinical trials on APL indicate that dose and schedule are critical, as is true for almost all pharmaceuticals. In addition, if one is aiming at modulating the immune response phenotype from $\mathrm{T}$ helper 1 to $\mathrm{T}$ helper 2, there may be associated allergic activity. Similar reactions at a similar frequency to those noted with the APL have been reported with Glatiramer actetate. ${ }^{9}$ Glatiramer acetate is known to modulate a T helper 2 response. ${ }^{9}$

\section{TRIALS WITH NATIVE MYELIN PROTEINS AND NATIVE MYELIN PEPTIDES}

Trials in the 1990s attempting to tolerize the immune system to myelin derived from a bovine source, showed promise in pilot studies. However, phase 3 trials failed to achieve their primary endpoints. In the pre-clinical trials investigators were able to show that oral adminstration of myelin induced circulating myelin basic protein and proteolipid protein $\mathrm{Th} 3 \mathrm{~T}$ cells capable of secreting transforming growth factor-Beta $1 .^{14}$ 
The major immunodominant $\mathrm{T}$ and $\mathrm{B}$ cell epitope for myelin basic protein lies between residues 82 and $99 .^{22,23}$ Intravenous administration of injection of myelin basic protein peptide 82-98, containing the immunodominant region VVHFFKNIVT, in a phase 1 trial lead to tolerance, with the absence of antibodies to myelin basic protein in the spinal fluid for up to 4 months. Tolerance was more enduring after a second injection. The effect was most pronounced in patients who were HLA DR2. ${ }^{30}$ In a Phase 2 trial with every-6-month injections, 32 patients with relapsing remitting MS were followed for 24 months. The major end point followed was changes in the expanded disability status scale EDSS. There were no significant changes in the whole cohort, but a subset of patients who were HLA DR2 and or DR4 showed a significant benefit in terms of EDSS score. No results on magnetic resonance imaging were reported. ${ }^{10}$ "Longterm follow-up treatment and assessment of patients in this responder group showed a median time to progression of 78 months for MBP8298 treated patients, compared with 18 months for placebo-treatment (KaplanMeier analysis, $\mathrm{P}=0.004$; relative rate of progression $=$ 0.23). Anti-MBP autoantibody levels in the CSF of most MBP8298-treated patients were suppressed, but antibody suppression was not predictive of clinical benefit." 10

\section{DNA VACCINATION TO MYELIN BASIC PROTEIN}

Pre-clinical studies have shown that it is possible to tolerize to myelin proteins using DNA vaccines incorporating full-length cDNAs encoding these proteins. Such trials showed that it was possible to reverse ongoing EAE, with decreased relapse frequency and a reduction in the spread of autoantibody responses to myelin. A 30-patient Phase 1 trial on individuals with relapsing remitting MS, was attempted with a DNA vaccine encoding full-length myelin basic protein. The main proinflammatory sequences in the non-coding region of the plasmid backbone, so called $\mathrm{CpG}$ sequences, were deleted and largely replacd with an anti-inflammatory sequence, called a GpG motif. ${ }^{11}$ These trials revealed a marked decrease in proliferation of interferon-gamma producing myelin-reactive $\mathrm{CD} 4+\mathrm{T}$ cells from peripheral blood, and a reduction in titers of myelin specific autoantibodies from cerebral spinal fluid as assessed by protein microarrays. ${ }^{11}$ A 300 -patient Phase 2 trial is nearly complete at the time of this submission.

\section{CONCLUSION}

At present, the neurological community is developing drugs that target wide swathes of the immune system. Very few efforts have been devoted to identifying those elements of the CNS that are targeted by adaptive im- munity in MS. Fewer efforts have aimed at tolerizing the immune system to those deleterious responses, while leaving the rest of the system intact. Some day we will be able to tolerize the adaptive immune system and abrogate only those responses that are involved in the pathology of MS. Currently, the major achievement in therapy of autoimmune diseases has been the use of anti-TNF for rheumatoid arthritis and Crohn's Disease. Other than the risk of opportunistic infections with tuberculosis, this approach has been a remarkable therapy for about twothirds of those with rheumatoid arthritis and with Crohn's. ${ }^{6}$ Unfortunately anti-TNF carries warnings in its label for worsening MS, so it is not a viable approach to demyelinating diseases. ${ }^{6}$ Therapy with antibody to alpha4 beta 1 integrin has proven to be a double-edged sword, providing great benefit but posing the risk of fatal infection with JC virus. ${ }^{4}$ The importance of developing antigen-specific therapy for MS is thus clear: Leaving the rest of the immune system intact to fight microbes and engage in surveillance against cancer, while tolerizing to those responses that are involved in MS is achievable, and worthy of more intensive efforts.

\section{REFERENCES}

1. Frohmann EM, Racke MK, Raine CS. Multiple sclerosis-the plaque and its pathogenesis. N Engl J Med 2006;354:942-955.

2. Steinman L. Multiple sclerosis: a two-stage disease. Nat Immunol 2001;2:762-764.

3. Weber M, Prod'homme T, Youssef S, et al. Type II monocytes modulate $\mathrm{T}$ cell-mediated central nervous system autoimmune disease. Nat Med 2007;13:935-943.

4. Steinman L. Blocking adhesion molecules as therapy for multiple sclerosis: natalizumab. Nat Rev Drug Discov 2005;4:510-518.

5. U.S. Food and Drug Administraion. Life-threatening brain infection in patients with systemic lupus erythematosus after Rituxan (rituximab) treatment [Internet]. FDA Public Health Advisory. December 18, 2006. Available at: http://www.fda.gov/cder/drug/advisory/rituximab.htm.

6. Feldmann M, Steinman L. Design of effective immunotherapy for human autoimmunity. Nature 2005;435:612-619.

7. Witkop B. Paul Ehrlich and his magic bullets: revisited. Proc Am Philos Soc 1999;143: 540-557.

8. Bielekova B, Goodwin B, Richert N, et al. Encephalitogenic potential of the myelin basic protein peptide (amino acids 83-99) in multiple sclerosis: results of a phase II clinical trial with an altered peptide ligand. Nat Med 2000;6:1167-1175.

9. Kappos L, Comi G, Panitch H, et al.; The Altered Peptide Ligand in Relapsing MS Study Group. Induction of a non-encephalitogenic type $2 \mathrm{~T}$ helper-cell autoimmune response in multiple sclerosis after administration of an altered peptide ligand in a placebo controlled, randomized phase II trial. Nat Med 2000;6:1176-1182 [Erratum in: Nat Med 2001;7:129].

10. Warren KG, Catz I, Ferenczi LZ, Krantz MJ.Intravenous synthetic peptide MBP8298 delayed disease progression in an HLA Class II-defined cohort of patients with progressive multiple sclerosis: results of a 24-month double-blind placebo-controlled clinical trial and 5 years of follow-up treatment. Eur J Neurol 2006;13:887895 .

11. Bar-Or A, Vollmer T, Antel J, et al. Induction of antigen-specific tolerance in multiple sclerosis after immunization with DNA encoding myelin basic protein in a randomized, placebo-controlled phase I/II trial. Arch Neurol 2007 Aug 13; [Epub ahead of print].

12. Salk, RJS. A study of myelin basic protein as a therapeutic probe in patients with multiple sclerosis. In: Hallpike JF, Adams CWM, 
Tourtellotte WW, editors. Multiple sclerosis: pathology, diagnosis, and management. London: Chapman and Hall, 1983:621-630.

13. Campbell B, Vogel PJ, Fisher E, Lorenz R. Myelin basic protein administration in multiple sclerosis. Arch Neurol 1973;29:10-15.

14. Faria AM, Weiner HL. Oral tolerance. Immunol Rev 2005;206: 232-259.

15. Steinman L. The coming of age for antigen specific therapy of multiple sclerosis. Eur J Neurol 2006;13:793-794.

16. van Noort JM, van Sechel AC, Bajramovic JJ, et al. The small heat-shock protein $\alpha \mathrm{B}$-crystallin as candidate autoantigen in multiple sclerosis. Nature 1995;375:798-801.

17. Ousman SS, Tomooka BH, Van Noort JM, et al. Protective and therapeutic role for $\alpha \mathrm{B}$-crystallin in autoimmune demyelination. Nature 2007;448:474-479.

18. Chabas D, Baranzini S, Mitchell D, et al. The influence of the pro-inflammatory cytokine, osteopontin, on autoimmune demyelinating disease. Science 2001;294:1731-1735.

19. Robinson WH, Fontoura P, Lee BJ, et al. Protein microarrays guide tolerizing DNA vaccine treatment of autoimmune encephalomyelitis. Nat Biotechnol 2003;21:1033-1039.

20. Banki K, Colombo E, Sia F, et al. Oligodendrocyte-specific expression and autoantigenicity of transaldolase in multiple sclerosis. J Exp Med 1994;180:1649-1663.

21. Kanter JL, Narayana S, Ho PP, et al. Lipid microarrays identify key mediators of autoimmune brain inflammation. Nat Med 2006; 12:138-143.

22. Sakai K, Zamvil SS, Mitchell DJ, Lim M, Rothbard JB, Steinman L. Characterization of a major encephalitogenic T cell epitope in $\mathrm{SJL} / \mathrm{J}$ mice with synthetic oligopeptides of myelin basic protein. J Neuroimmunol 1988;19:21-32.

23. Warren KG, Catz I, Steinman L. Fine specificity of the antibody response to myelin basic protein in the central nervous system in multiple sclerosis: the minimal B-cell epitope and a model of its features. Proc Natl Acad Sci U S A 1995;92:11061-11065.

24. Wucherpfennig KW, Catz I, Hausmann S, Strominger JL, Steinman L, Warren KG. Recognition of the immunodominant myelin basic protein peptide by autoantibodies and HLA-DR2-restricted T cell clones from multiple sclerosis patients: identity of key contact residues in the B-cell and T-cell epitopes. J Clin Invest 1997;100: $1114-1122$.

25. Karin N, Mitchell DJ, Brocke S, Ling N, Steinman L. Reversal of experimental autoimmune encephalomyelitis by a soluble variant of a myelin basic protein epitope: $\mathrm{T}$ cell receptor antagonism and reduction of interferon gamma and tumor necrosis factor alpha production. J Exp Med 1994;180:2227-2237.

26. Brocke S, Gijbels K, Allegretta M, et al. Treatment of experimental encephalomyelitis with a peptide analogue of myelin basic protein. Nature 1996;379:343-345.

27. Gaur A, Boehme SA, Chalmers D, et al. Amelioration of relapsing experimental autoimmune encephalomyelitis with altered myelin basic protein peptides involves different cellular mechanisms. J Neuroimmunol 1997;74:149-158.

28. Crowe PD, Qin Y, Conlon PJ, Antel JP. NBI-5788, an altered MBP83-99 peptide, induces a T-helper 2-like immune response in multiple sclerosis patients. Ann Neurol 2000;48:758-765.

29. Kim HJ, Antel JP, Duquette P, Alleva DG, Conlon PJ, Bar-Or A. Persistence of immune responses to altered and native myelin antigens in patients with multiple sclerosis treated with altered peptide ligand. Clin Immunol 2002;104:105-114.

30. Warren KG, Catz I, Wucherpfennig KW. Tolerance induction to myelin basic protein by intravenous synthetic peptides containing epitope P85 VVHFFKNIVTP96 in chronic progressive multiple sclerosis. J Neurol Sci 1997;152:31-38. 